Rapid Commun. Mass Spectrom. 2013, 27, 2310-2316

(wileyonlinelibrary.com) DOI: 10.1002/rcm.6697

\title{
Electrostatic-spray ionization mass spectrometry sniffing for perfume fingerprinting
}

\author{
Elena Tobolkina ${ }^{1}$, Liang Qiao ${ }^{1}$, Guobin $\mathrm{Xu}^{2}$ and Hubert H. Girault ${ }^{1 *}$ \\ 'Laboratoire d'Electrochimie Physique et Analytique, Ecole Polytechnique Fédérale de Lausanne (EPFL), Station 6, CH-1015, \\ Lausanne, Switzerland \\ ${ }^{2}$ Department of Chemistry and Institute of Biomedical Sciences, Fudan University, Shanghai 200433, P.R. China
}

RATIONALE: The perfume market is growing significantly, and it is easy to find imitative fragrances of probably all types of perfume. Such imitative fragrances are usually of lower quality than the authentic ones, creating a possible threat for perfume companies. Therefore, it is important to develop efficient chemical analysis techniques to screen rapidly perfume samples.

METHODS: Electrostatic-spray ionization (ESTASI) was used to analyze directly samples sprayed or deposited on different types of paper. A linear ion trap mass spectrometer was used to detect the ions produced by ESTASI with a modified extended transfer capillary for 'sniffing' ions from the paper.

RESULTS: Several commercial perfumes and a model perfume were analyzed by ESTASI-sniffing. The results obtained by paper ESTASI-MS of commercial fragrances were compared with those obtained from ESI-MS. In addition, a commercial fragrance was first nebulized on the hand and then soaked up by blotting paper, which was afterwards placed on an insulating plate for ESTASI-MS analysis. Analysis of peptides and proteins was also performed to show that the paper ESTASI-MS could be used for samples with very different molecular masses.

CONCLUSIONS: Paper ESTASI-MS yields a rapid fingerprinting characterization of perfume fragrances, avoiding timeconsuming sample-preparation steps, and thereby performing a rapid screening in a few seconds. Copyright $@ 2013$ John Wiley \& Sons, Ltd.

Mass spectrometry has been one of the most rapidly developing chemical analysis techniques over the last decades, applied in various research fields, including organic chemistry, polymer science, life science, etc. One major challenge in mass spectrometry is to directly analyze untreated samples in their ambient state, which calls for the development of new atmospheric ionization methods. ${ }^{[1]}$ Nowadays, techniques such as electrospray ionization mass spectrometry (ESI-MS) ${ }^{[2,3]}$ desorption electrospray ionization $(\mathrm{DESI})^{[1]} \mathrm{MS}$ and extractive electrospray ionization $(\mathrm{EESI})^{[4]}$ MS allow liquid samples to be identified and classified in a rapid online mode. ${ }^{[5]}$

We have recently developed an electrostatic-spray ionization (ESTASI) method, which, in positive ion mode, can generate protonated molecules from samples deposited as droplets on a plastic substrate or inside a polyacrylamide gel. ${ }^{[6,7]}$ Herein, ESTASI is further applied to samples on lint-free paper. A paper spray ionization method was introduced by Wang et al. in 2010, where samples were deposited on a piece of paper that was connected to a high voltage for electrospray ionization. ${ }^{[8]}$

* Correspondence to: H. H. Girault, Laboratoire d'Electrochimie Physique et Analytique, Ecole Polytechnique Fédérale de Lausanne (EPFL), Station 6, CH-1015 Lausanne, Switzerland. E-mail: hubert.girault@epfl.ch
This technique has since been applied for the qualitative and quantitative analysis of dried biofluid spots and drugs.

Different from the previously reported paper spray ionization approaches, in the ESTASI method the high voltage (HV) is not applied directly to the paper, but to an electrode that is isolated from the paper by a plastic plate (insulator) during paper ESTASI. The electrode is connected in turn to the HV and ground under the control of two switches to induce ESTASI under ambient conditions. When the electrode is connected to $\mathrm{HV}$, a spray of cations is produced during the charging of a capacitor formed by electrode, insulator and sample solution. When the electrode is at ground, a spray of anions is produced during the discharge of the capacitor. A modified 'sniffing' transfer capillary collects the generated ions for MS analysis. Paper ESTASI is here used for protein/peptide analysis and for the fast characterization of perfumes that are deposited or sprayed on a piece of lint-free paper.

Fine fragrances are mixtures of volatile synthetic chemicals as well as natural extracts such as essential oil and concentrates of flower, fruits, spices, etc., obtained by a wide range of methods such as expression, solvent extraction and steam distillation. ${ }^{[9]}$ Perfumes consist of typically 10 to 300 of such constituents, leading to very complex mixtures. ${ }^{[10]}$ Nevertheless, thanks to constant improvement in the speed and efficiency of analytical techniques, ${ }^{[11]}$ it is now possible to analyze the composition of perfumes, leading to the risk 
of counterfeit samples being produced for major perfume brands. Such counterfeit fragrances are usually of lower quality than the authentic ones, and represent a violation of the Intellectual Rights of the perfume producer, creating a possible threat for perfume companies. Therefore, it is important to develop efficient chemical analysis techniques for the rapid screening of perfume samples. ${ }^{[12]}$

Gas chromatography (GC) coupled with detection methods, such as flame ionization detection (FID) ${ }^{[13]}$ Fourier transform infrared spectroscopy (FT-IR), mass spectrometry (MS) ${ }^{[14-17]}$ and tandem mass spectrometry (MS/MS), ${ }^{[18]}$ has been the technique of choice for the qualitative and quantitative analyses of volatile and semi-volatile compounds in cosmetic products and perfume. ${ }^{[19]}$ However, these widely used methods suffer from some limitations: (i) sample preparation complexity, in particular where the cosmetics also contain non-volatile ingredients not compatible with GC/MS analysis, and (ii) the time required for analysis which prevents the study of large sets of samples. ${ }^{[20]}$ To circumvent these limitations, the development of new analytical methods for the screening of fragrances is needed.

Recently, EESI-MS was applied for the classification of perfumes as a high-throughput and sample-preparation-free method. ${ }^{[4]}$ ESI-MS in a standard infusion mode can also be applied in perfume analysis, but it is limited by the preparation procedures because of the strong adsorption of volatile molecules inside the syringe and the transfer capillary. Herein, we have demonstrated that paper ESTASI-MS is a technique that generates perfume mass spectra similar to those obtained using an electrospray ionization (ESI) source but without sample preparation, thus in a more convenient and faster manner. Six authentic fragrances and a model perfume prepared by mixing ten different compounds were used as samples for analysis. To assess the performance of this new experimental setup, analyses of peptides and proteins deposited on paper were first carried out.

\section{EXPERIMENTAL}

\section{Materials}

Angiotensin I ( $\left.\mathrm{NH}_{2}-\mathrm{DRVYIHPFHL}-\mathrm{COOH}, 98 \%\right)$ was obtained from Bachem (Basel, Switzerland). Cytochrome c from horse heart $(12.3 \mathrm{kDa})$ as well as methanol and acetic acid of the purest grade $(>99.9 \%)$ were obtained from Sigma-Aldrich (Schnelldorf, Switzerland). Deionized (DI) water was purified by an alpha Q system (Millipore, Zug, Switzerland).

A 'model perfume' sample consisting of ten different compounds (ambrox, citronellyl nitrile, cyclogalbanate, damascenone, diphenyl ether, hedione, neobutenone alpha, iso e super, sclareolate, tonalide pf) was prepared in our laboratory. The structures and molecular weights of these compounds are given in Supplementary Table S1 (see Supporting Information). Six authentic fragrances: 'Un Jardin sur le Nil' by Hermes, 'Flower' by Kenzo, 'Ange ou demon' by Givenchy, 'Encre Noire' by Lalique, 'Midnight in Paris' by Van Cleef \& Arpels, and 'The one gentleman' by Dolce \& Gabbana. Perfume testing strips and 'Chanel' blotting paper were obtained from local stores. Lint-free paper (Contec Wipe Nw CEL/POLY 9X9IN PK300 C1-99) was acquired from Contec (Toledo, $\mathrm{OH}, \mathrm{USA})$.

\section{Paper electrostatic-spray ionization}

A volume of $5 \mu \mathrm{L}$ of the sample containing either the peptide/protein in the acidic buffer (50\% methanol, $49 \%$ water and $1 \%$ acetic acid) or the perfume sample was deposited with a micropipette or nebulized directly from the perfume bottle on top of a paper strip $(6 \mathrm{~cm}$ long, $2 \mathrm{~cm}$ wide), which was placed under the modified mass spectrometer inlet. The paper strip here can be the fragrance testing strip, the 'Chanel' blotting paper or the lint-free paper. An electrode was placed behind and isolated from the paper by a piece of plastic, and facing the sample to induce electrostatic-spray. The electrode was connected either to a direct current (DC) high-voltage $(9 \mathrm{kV})$ source or to ground using two switches. A special LabView programme was written to control the switches in order to synchronize their work. ${ }^{[6]}$

\section{Mass spectrometer}

A linear ion trap Thermo LTQ Velos (Thermo Scientific, Reinach, Switzerland) mass spectrometer was used to detect the ions produced by ESTASI with a modified extended transfer capillary for 'sniffing' ions from the paper. The electrospray voltage of the LTQ Velos was set at 0. A highvoltage battery as an external power source was used for the ESTASI. An enhanced ion trap scanning rate $(10000 \mathrm{~m} / \mathrm{z}$ units/s) was used for the MS analysis. All the spectra were recorded in positive ion mode.

\section{RESULTS AND DISCUSSION}

\section{Paper electrostatic-spray ionization mass spectrometry}

To characterize the perfume from the lint-free paper, the electrostatic spray ionization (ESTASI) method was used. ${ }^{[6,7]}$ In simple terms, a sample solution is placed on an inert insulating support placed in front of the mass spectrometer inlet. An electrode is placed behind the support and is connected to a pulsed high-voltage source. When a positive high voltage is applied, charge separation occurs in the sample solution, and the sample-air interface becomes polarized. When the charge accumulated is large enough for the electrostatic pressure to be larger than the Laplace pressure, electrospray of cations towards the mass spectrometer inlet takes place, leaving net negative charges in the sample solution. By grounding the electrode, anions will again charge the liquid-air interface, leading to electrospray of anions.

In a previous publication, ESTASI of a sample solution from a microchannel, a capillary, and droplets on a plastic plate was reported. ${ }^{[6]}$ Here, we demonstrate that ESTASI can also be used for samples within a piece of lint-free paper that is placed on a plastic plate, as shown in Scheme 1. The sample solution was deposited on top of the paper and quickly adsorbed by the fibers of the paper, without forming droplets or only forming a thin layer of liquid. The paper strip was placed on the insulating plate between the electrode and mass spectrometer inlet, where the plate can move in $x, y$ directions in order to align the sample right under and close enough to the mass spectrometer inlet. A high potential of $9.0 \mathrm{kV}$ was used to induce ESTASI. By applying a pulsed high 


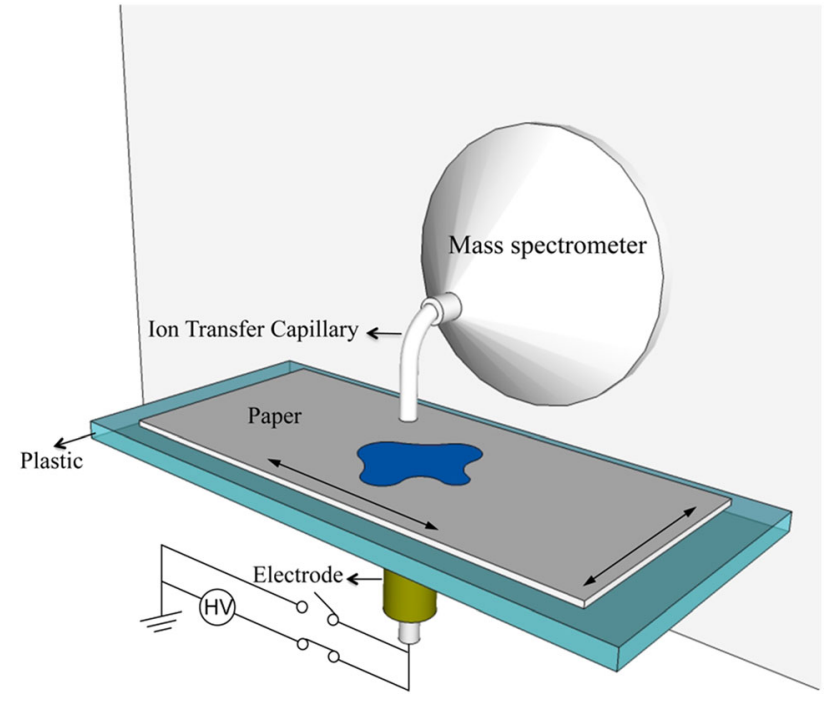

Scheme 1. Schematic representation of paper ESTASI-MS with modified ion transfer capillary.

voltage to the electrode, samples were ionized for MS detection before the solvent was completely evaporated from the paper. The ion transfer capillary of the mass spectrometer was modified as shown in Scheme 1 to 'sniff' the ions from the paper, which was conveniently achieved by scanning a horizontal surface plate placed in front of the mass spectrometer inlet.

To test this new experimental setup, different experiments were first carried out on model samples. First, paper ESTASI was performed with the angiotensin I peptide $\left(\mathrm{NH}_{2}\right.$-DRVYIHPFHL-COOH) at different concentrations in an acid buffer (50\% methanol, $49 \%$ water and $1 \%$ acetic acid).
The sample $(5 \mu \mathrm{L})$ was deposited on top of the testing strip and a high voltage of $9.0 \mathrm{kV}$ was used for ESTASI. During ESTASI, no droplets were formed. The spray occurred either from a thin and flat layer of liquid or from the solution in the structure of the paper that can be viewed as arrays of micropores. As soon as the positive high voltage was applied to the electrode, cations were generated by ESTASI for MS detection. As shown in Fig. 1, the peptide was easily detected by paper ESTASI-MS, and the limit of detection (LOD) of the peptide was found to be $\sim 250 \mathrm{nM}$. Cytochrome c protein in ESI buffer was analyzed in a similar way by paper ESTASIMS. As shown in Fig. 1, this protein can be easily detected, with a LOD of $1.6 \mu \mathrm{M}$.

\section{Paper ESTASI for the analysis of a model perfume}

Perfume samples may contain hundreds of different constituents. It is quite difficult to detect all of them using mass spectrometry, as not all the compounds from the sample can be ionized by electrospray ionization. A 'model perfume' sample was used as a standard sample to demonstrate the concept of using paper ESTASI-MS for perfume analysis. The model perfume was prepared by mixing ten compounds in different ratios.

The model perfume was first analyzed by ESI-MS using the instrument's ESI source in direct-infusion and positive ion mode. The mass spectrum obtained is shown as Fig. 2(a). Five of the ten compounds from the mixture were detected and identified, while the others could not be ionized by ESI to be detected. Some peaks in the ESI mass spectrum could not be identified as components of the model perfume and might be attributed to products of chemical or electrochemical reactions during the ESI process or as the background peaks in the instrument. Although a good ESI mass spectrum for
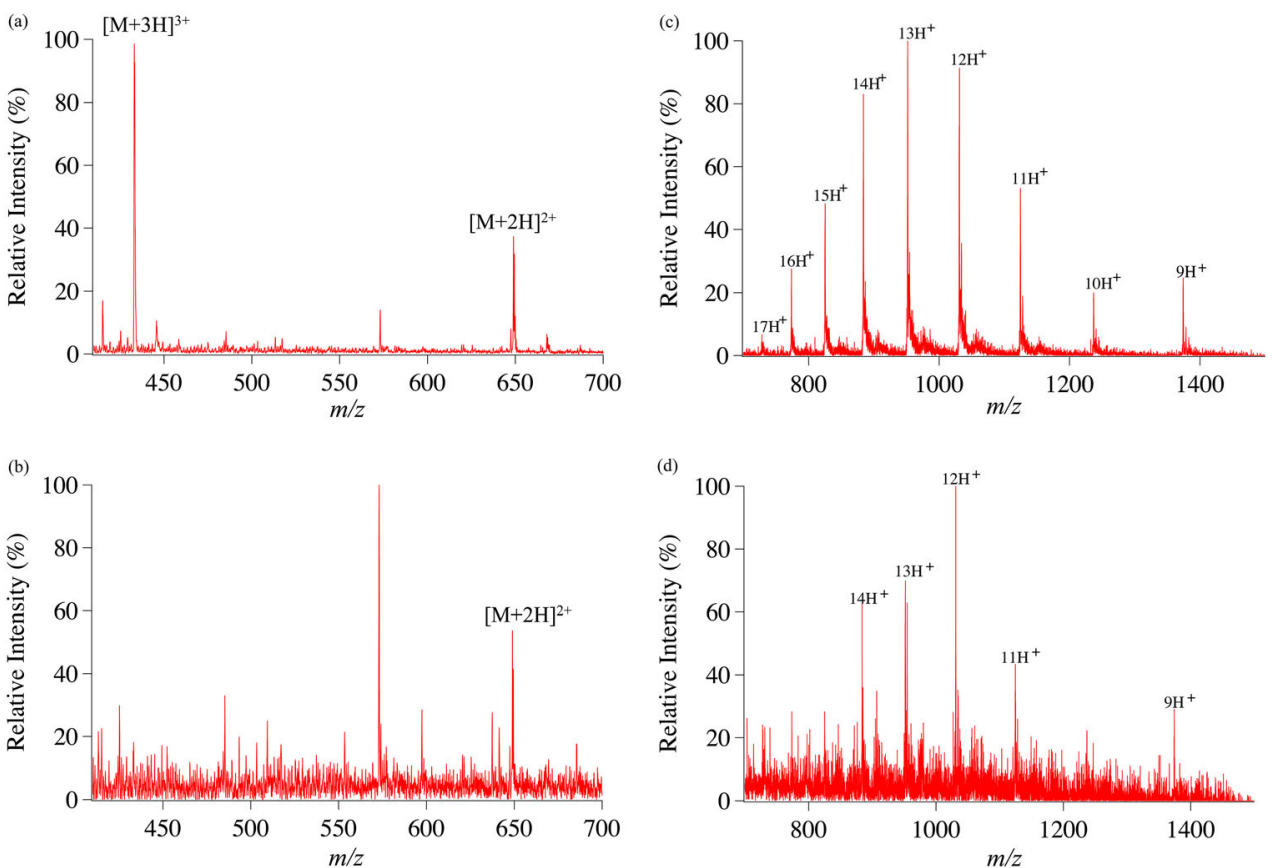

Figure 1. Mass spectra of: (a) $25 \mu \mathrm{M}$ of angiotensin I, (b) $250 \mathrm{nM}$ of angiotensin I, (c) $16 \mu \mathrm{M}$ of cytochrome c, and (d) $1.6 \mu \mathrm{M}$ of cytochrome c sprayed from the paper strip by ESTASI. All the mass spectra were recorded in positive MS mode. The ions were generated by paper ESTASI when a pulsed positive high potential $(9.0 \mathrm{kV})$ was applied to the electrode. 

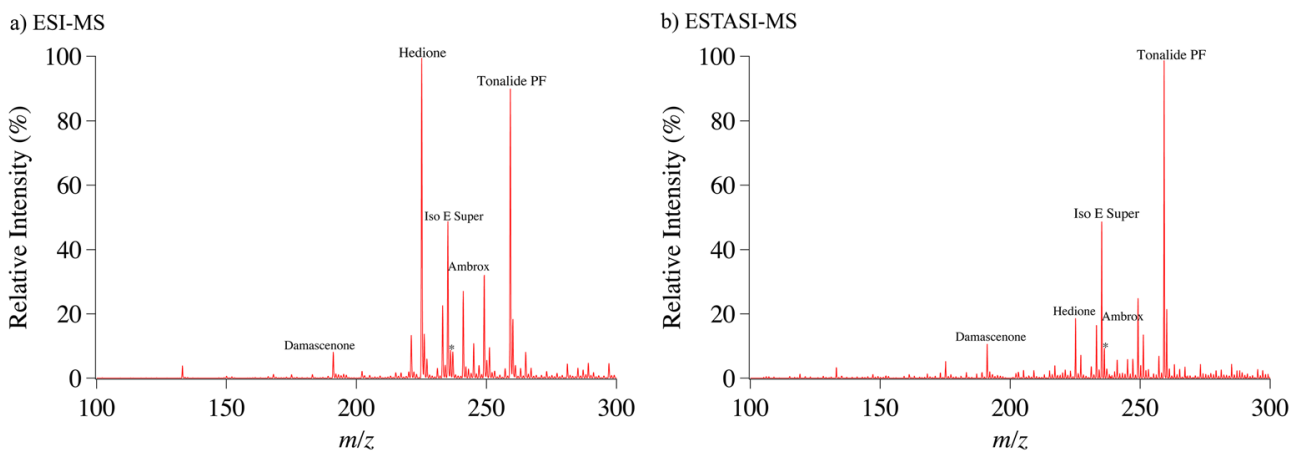

Figure 2. Mass spectra of the model perfume sample obtained by: (a) ESI-MS and (b) ESTASI-MS in positive ion mode. The labeled peaks correspond to singly protonated molecules.
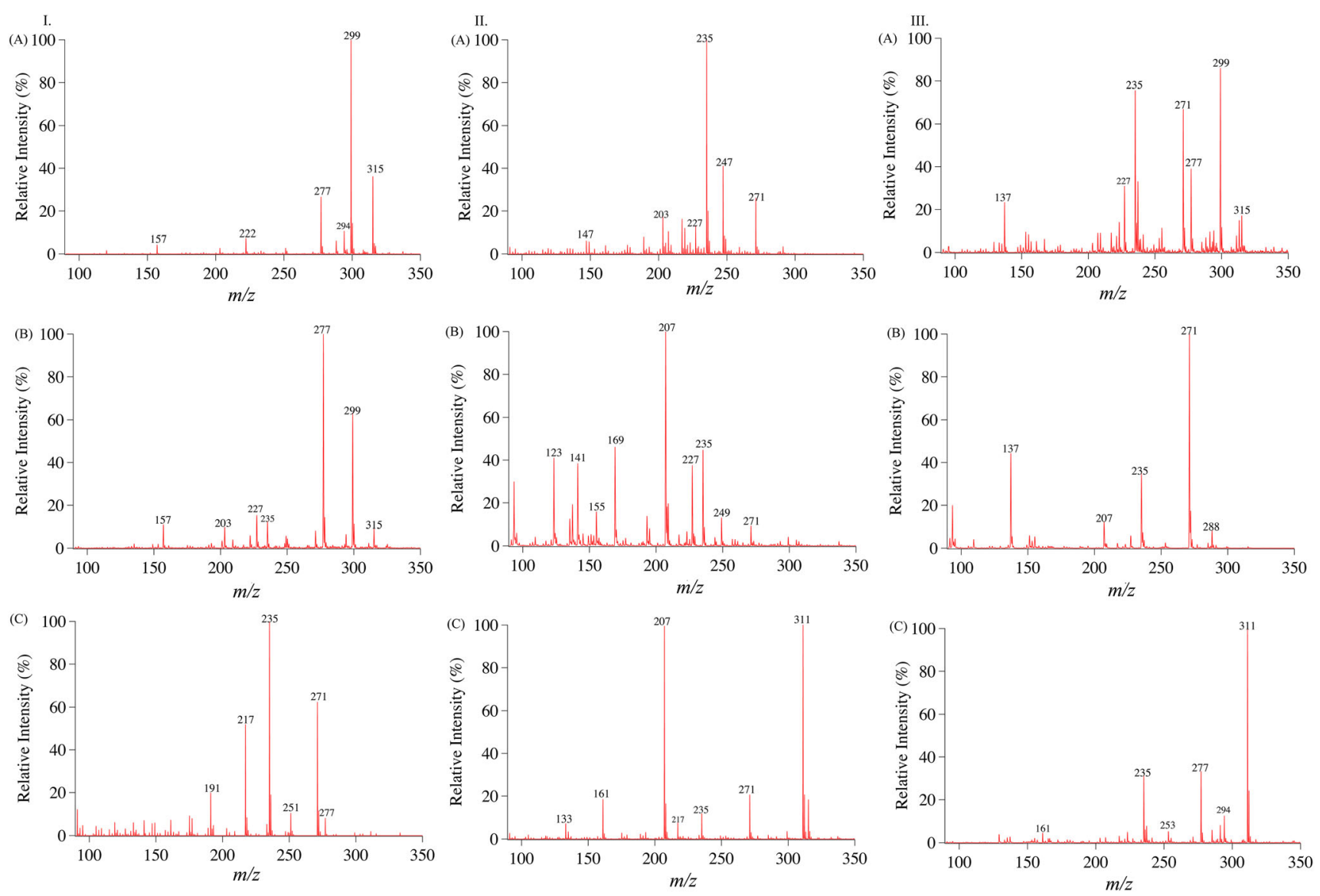

Figure 3. Mass spectra of three authentic fragrances (I. 'Ange ou demon' by Givenchy; II. 'Un Jardin sur le Nil' by Hermes; III. 'Flower' by Kenzo) obtained by (A) ESI with standard ESI source in direct-infusion mode, (B) paper ESTASI-MS with the substrate of lint-free paper, and (C) paper ESTASI-MS with the substrate of perfume testing strip from the local shop. The MS experiments were performed in positive ion mode.

the model perfume was obtained with the instrument's ESI source in direct-infusion mode, this is not an ideal technique for the high-throughput analysis of perfume. One problem is that the volatile compounds in the perfume are easily adsorbed as contaminants on the wall of the polymer capillary that is used to transfer sample solution from the syringe to the ESI source. It took hours of washing of the capillary to completely remove the adsorbed molecules and recover a clean background.
Paper ESTASI-MS was used to avoid this cleaning step. The model perfume was deposited with a micropipette on top of the lint-free paper just under the mass spectrometer inlet. By applying the high voltage $(9 \mathrm{kV})$, the sample was sprayed from the surface of the paper and directly analyzed by MS in positive ion mode. As shown in Fig. 2(b), the same five compounds as found by conventional ESI-MS were identified by paper ESTASI-MS. Since the lint-free paper strip is disposable, the cleaning steps are avoided and no contamination from strip- 
Table 1. Peak list of ESI-MS and ESTASI-MS with the lintfree paper, blotting paper and the fragrance testing strip of the 'Givenchy' perfume (+ observed; - not observed)

\section{$m / z$ ESI-MS Lint-free paper Testing strip Blotting paper}

\begin{tabular}{|lllll|}
\hline 157 & + & + & - & - \\
161 & - & - & + & - \\
175 & - & - & + & - \\
191 & - & - & + & + \\
203 & + & + & - & - \\
209 & - & + & - & - \\
222 & + & + & + & + \\
227 & - & + & - & - \\
235 & - & + & + & + \\
251 & + & + & + & + \\
271 & - & + & + & + \\
277 & + & + & - & - \\
294 & + & + & - & + \\
299 & + & + & - & + \\
315 & + & + & & + \\
\hline
\end{tabular}

to-strip was observable. Negative ion mode was also tested, but did not give any identification of the perfume compounds with either ESI-MS or ESTASI-MS, because none of the compounds could be easily deprotonated.

\section{Paper ESTASI for the analysis of six authentic fragrances}

The paper ESTASI was further applied for the fast analysis of real complex perfume samples. Six authentic fragrances including three women's and three men's were analyzed. Each fragrance was deposited $(5 \mu \mathrm{L})$ by a micropipette or nebulized on top of a paper strip, which was then placed under the ion transfer capillary. ESTASI was performed from the region where the sample was loaded and the compounds of the perfume were extracted and detected by MS. Figure 3 shows the paper ESTASI-MS results of three women's perfume fragrances ('Givenchy', 'Hermes', 'Kenzo'), which were obtained with the different types of paper strips. ESI-MS was also performed for all the fragrances with the commercial source mounted on the LTQ Velos for comparison.

It can be clearly seen that the most abundant peaks appear in the $m / z$ 100-300 range, corresponding to highly volatile compounds. The results obtained by ESI-MS of each fragrance were compared with those obtained from paper ESTASI-MS with the lint-free or the perfume testing strip. The abundances of the peaks of the different ions detected depended on the spray surface, perhaps because the adsorption of the perfume compounds by different types of paper is different (see Figs. 3 and 5). As shown in Fig. 3I and Table 1, ESTASI-MS from lint-free paper gave the most similar peaks from the 'Givenchy' perfume to those obtained by ESI-MS. The peaks at $m / z$ 157, 222, 277, 299 and 315 were abundant and observed by both ESI and paper ESTASI with lint-free paper. However, the paper ESTASI of this perfume from the perfume testing strip also showed similar results to those obtained by ESI-MS.

Before the analyses of the commercial fragrances from the paper, a blank analysis was performed by ESTASI-MS. Several microliters of ESI buffer were deposited on the paper strip and the high voltage was applied. None of the peaks detected from the perfume fragrances were observed during the 'blank' experiment.

Figure 4 represents the steps of depositing and analyzing the 'Givenchy' fragrance from the blotting paper by ESTASI-MS. The sample was first nebulized on the skin from the original bottle and then soaked by the blotting paper, which was afterwards placed on the insulating plate for ESTASI-MS analysis. As shown in Fig. 4 and Table 1, the blotting method also gave a detection of compounds similar to that obtained by ESI-MS.

As shown in Figs. 3II and III, the compounds detected by ESI-MS and paper ESTASI-MS with different substrates share some similarities but also show some differences. With the current methods, of course it is not possible to identify each observed peak and it is not possible to observe most compounds from each perfume. However, it is possible to use the spectra as chemical fingerprints for the perfumes since the spectra of the different perfumes are highly different and the spectra for each perfume are reproducible. Combining the spectra obtained with different paper substrates, such chemical fingerprints for perfumes can be good identities.

Three men's perfumes were also analyzed and compared by ESI-MS, paper ESTASI-MS with lint-free paper and paper ESTASI-MS with the fragrance testing strip. As shown in Fig. 5, the most abundant peaks also appear in the $m / z 100-300$ ranges, corresponding to the volatile components of the perfume that could be easily desorbed from the surface of the paper strip. The spectra of the different perfumes obtained with the same method again look different, further suggesting these spectra as chemical fingerprints of perfumes.

The most abundant peaks observed from the different perfumes by paper ESTASI-MS with lint-free paper as substrate are listed in Table 2. The peak lists of the women's fragrances are not very different from those of the men's. The ions at $m / z 137,157$ correspond to protonated limonene and citronellol that can be found in the ingredients list for all the perfumes. The peak list obtained for all the perfume fragrances looks similar to each other, suggesting that some
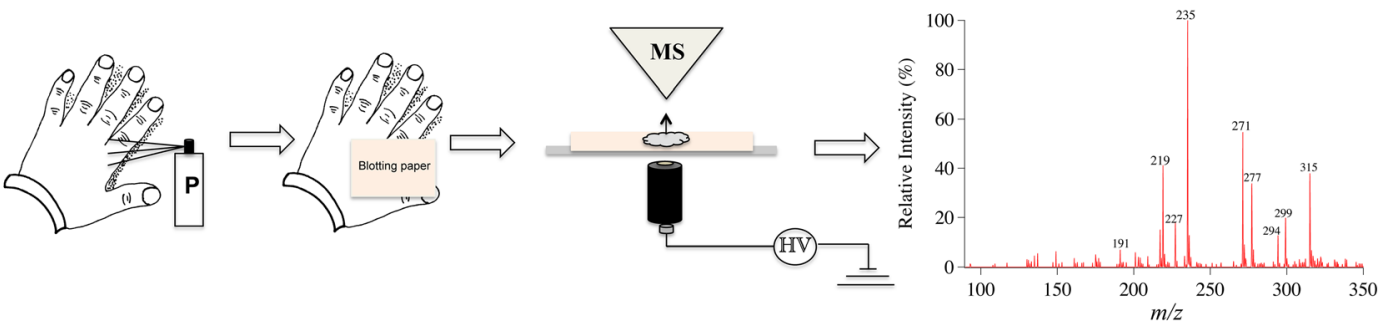

Figure 4. 'Givenchy' perfume nebulization on the hand, soaking the perfume with a blotting paper from the hand and paper ESTASI-MS analysis, P: perfume, MS: mass spectrometry analysis. 

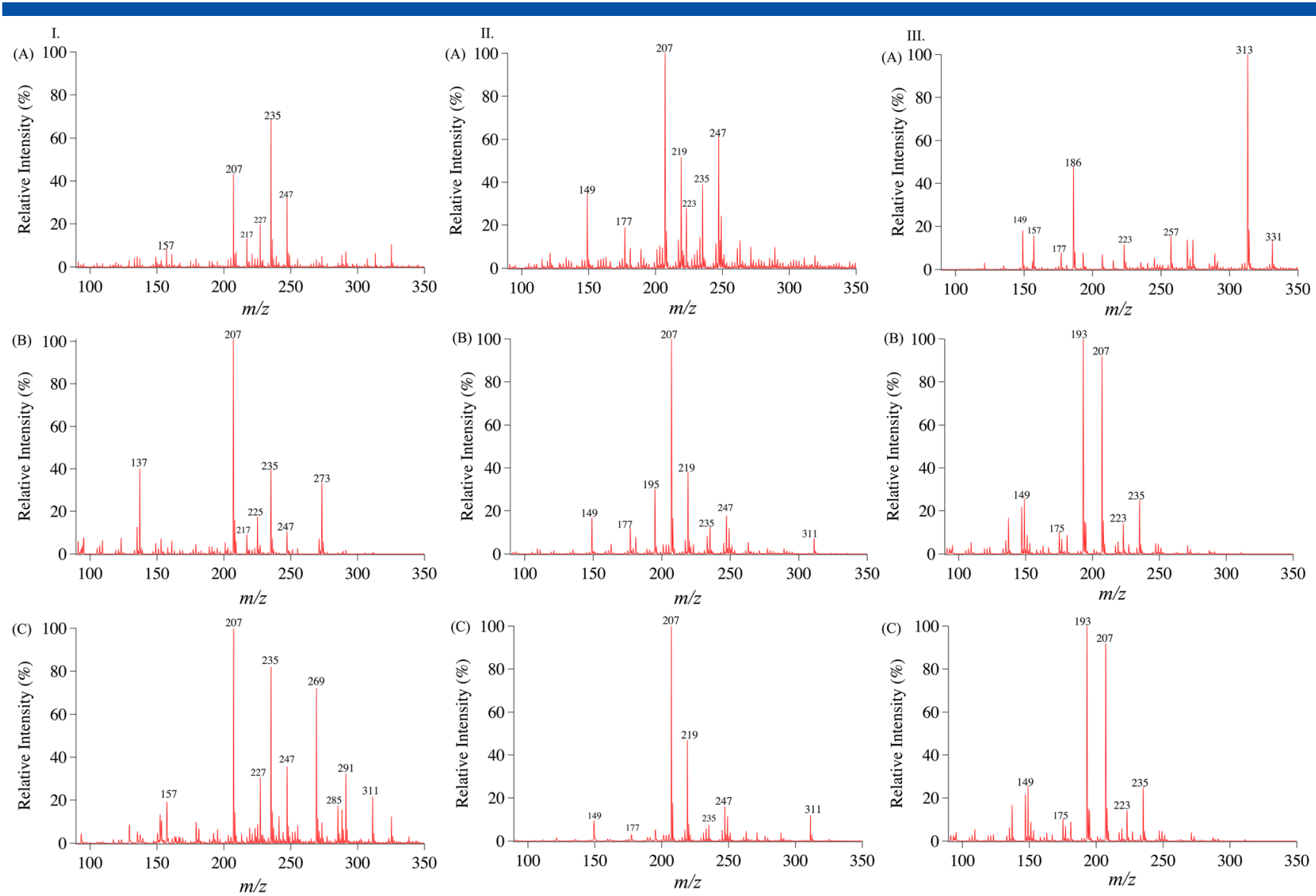

Figure 5. Mass spectra of the three men's authentic fragrances (I. 'The one gentleman' by Dolce \& Gabbana; II. 'Encre Noire' by Lalique and III. 'Midnight in Paris' by Van Cleef \& Arpels) obtained by electrospray ionization (A) and paper ESTASI-MS with the substrate of lint-free paper (B), and perfume testing strip (C) from the local shop. The spectra were obtained in positive ion mode.

Table 2. Peak list of the 11 most abundant peaks of the 'Hermes', 'Kenzo', 'Dolce \& Gabbana' (D\&G), 'Lalique' and 'Van Cleef \& Arpels' (VCA) perfume obtained from the lint-free paper by ESTASI-MS

\begin{tabular}{|lccccc|}
\hline $\boldsymbol{m} / \boldsymbol{z}$ & Hermes & Kenzo & D\&G & Lalique & VCA \\
\hline 1 & 135 & 137 & 135 & 149 & 137 \\
2 & 137 & 151 & 137 & 163 & 149 \\
3 & 141 & 207 & 149 & 177 & 175 \\
4 & 155 & 227 & 157 & 181 & 181 \\
5 & 169 & 235 & 161 & 195 & 193 \\
6 & 193 & 253 & 207 & 207 & 207 \\
7 & 207 & 271 & 217 & 219 & 219 \\
8 & 227 & 277 & 227 & 235 & 223 \\
9 & 235 & 288 & 235 & 247 & 235 \\
10 & 249 & 299 & 247 & 263 & 247 \\
11 & 271 & 311 & 273 & 311 & 271 \\
\hline
\end{tabular}

of the same compounds are used for perfume preparation for both women's and men's fragrances. However, in all commercial fragrances there are some specific peaks, which show the difference between the perfumes. The peaks with $m / z$ 137, 207 and 235 are present in several fragrances. The ion with $m / z 151$ was observed only in 'Kenzo', $m / z 263$ in 'Lalique', $m / z 141$ in 'Hermes', $m / z 273$ in 'D\&G' and

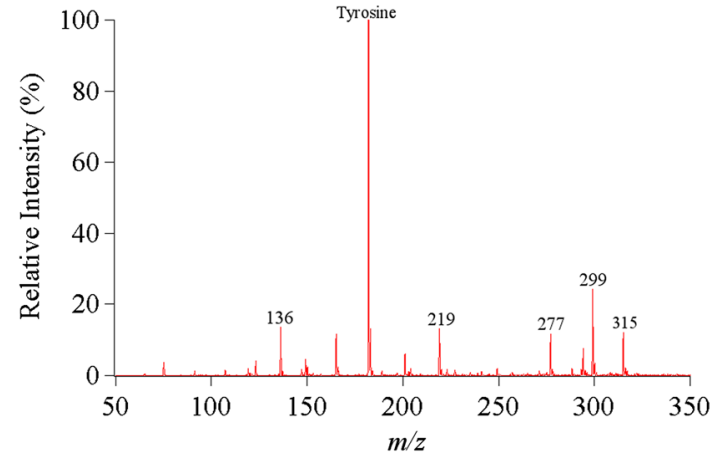

Figure 6. Mass spectra of Ange ou demon' by Givenchy mixed with $10 \mathrm{mM}$ tyrosine solution. The MS data was obtained in positive ion mode.

$m / z 223$ in 'VCA'. The perfumes are complex mixtures, containing not only chemicals giving odor, but also compounds for stabilization and dispersion, resulting in the different observed peaks.

Non-volatile and odorless samples that produce ions with $m / z$ values in the range of 100-300 can also be detected by paper ESTASI-MS together with the perfume compounds. Indeed, these non-volatile and odorless samples (an amino acid in our case) may be used as security labels for the 
perfume products to distinguish them from the counterfeits. To demonstrate this concept, tyrosine was mixed with the 'Givenchy' original fragrance and $5 \mu \mathrm{L}$ of the solution was deposited on top of the lint-free paper. After applying the high voltage the main peak, $m / z 182$ of protonated tyrosine, and the main peaks of the fragrance were observed by MS (see Fig. 6).

\section{CONCLUSIONS}

We have developed a strategy of rapid analysis of perfume fragrances by paper-based electrostatic-spray ionization mass spectrometry. A modified ion transfer capillary was used to simplify the ion detection procedure. The spectra from paper ESTASI-MS can be used as chemical fingerprints of perfumes. As an ambient ionization method for untreated samples, paper ESTASI-MS is a high-throughput method for perfume analysis, compared with direct-infusion ESI-MS, as it avoids time-consuming cleaning steps. With these proof-of-concept results, we could expect this method to be applied in numerous research fields, such as quality control of perfume fragrances, aromatic and natural compounds, increasing the productivity of the perfume and cosmetic industry.

\section{SUPPORTING INFORMATION}

Additional supporting information may be found in the online version of this article.

\section{Acknowledgement}

The authors would like to thank Dr Laurent Wunsche from Firmenich International SA, Switzerland, for helpful suggestions and discussions.

\section{REFERENCES}

[1] R. G. Cooks, Z. Ouyang, Z. Takats, J. M. Wiseman. Ambient mass spectrometry. Science 2006, 311, 1566.

[2] M. Yamashita, J. B. Fenn. Electrospray ion-source - Another variation on the free-jet theme. J. Phys. Chem. 1984, 88, 4451.

[3] J. B. Fenn, M. Mann, C. K. Meng, S. F. Wong, C. M. Whitehouse. Electrospray ionization for mass-spectrometry of large biomolecules. Science 1989, 246, 64.

[4] K. Chingin, G. Gamez, H. W. Chen, L. Zhu, R. Zenobi. Rapid classification of perfumes by extractive electrospray ionization mass spectrometry (EESI-MS). Rapid Commun. Mass Spectrom. 2008, 22, 2009.

[5] O. P. Haefliger, N. Jeckelmann. Direct mass spectrometric analysis of flavors and fragrances in real applications using DART. Rapid Commun. Mass Spectrom. 2007, 21, 1361.

[6] L. Qiao, R. Sartor, N. Gasilova, Y. Lu, E. Tobolkina, B. H. Liu, H. H. Girault. Electrostatic-spray ionization mass spectrometry. Anal. Chem. 2012, 84, 7422.

[7] E. Tobolkina, L. Qiao, L. Baohong, H. Girault. Coupling isoelectric focusing gel electrophoresis to mass spectrometry by electrostatic spray ionization. Anal. Chem. 2013, 85, 4745.

[8] H. Wang, J. Liu, R. G. Cooks, Z. Ouyang. Paper spray for direct analysis of complex mixtures using mass spectrometry. Angew. Chem. Int. Ed. 2010, 49, 877.

[9] A. D. Fortineau. Chemistry perfumes your daily life. J. Chem. Ed. 2004, 81, 45

[10] S. C. Rastogi, J. P. Lepoittevin, J. D. Johansen, P. J. Frosch, T. Menne, M. Bruze, B. Dreier, K. E. Andersen, I. R. White. Fragrances and other materials in deodorants: search for potentially sensitizing molecules using combined GC-MS and structure activity relationship (SAR) analysis. Contact Dermatitis 1998, 39, 293.

[11] A. van Asten. The importance of GC and GC-MS in perfume analysis. Trac - Trends Anal. Chem. 2002, 21, 698.

[12] C. Bicchi, P. Rubiolo, C. Cordero. Separation science in perfume analysis. Anal. Bioanal. Chem. 2006, 384, 53.

[13] L. Mondello, A. Casilli, P. Q. Tranchida, G. Dugo, P. Dugo. Comprehensive two-dimensional gas chromatography in combination with rapid scanning quadrupole mass spectrometry in perfume analysis. J. Chromatogr. A 2005, 1067, 235.

[14] M. Chien. Analysis of complex-mixtures by gaschromatography mass-spectrometry using a patternrecognition method. Anal. Chem. 1985, 57, 348.

[15] S. Rastogi. Analysis of fragrances in cosmetics by gas chromatography-mass spectrometry. J. High Res. Chromatogr. 1995, 18, 653.

[16] H. Casabianca, J. B. Graff, P. Jame, C. Perrucchietti, M. Chastrette. Application of hyphenated techniques to the chromatographic authentication of flavors in foodproducts and perfumes. Hrc-J. High Res. Chromatogr. 1995, 18, 279.

[17] M. Lopez-Nogueroles, J. L. Benedé, A. Chisvert, A. Salvador. A rapid and sensitive gas chromatography-mass spectrometry method for the quality control of perfumes: simultaneous determination of phthalates. Anal. Methods 2013, 5, 409.

[18] M. Jalali-Heravi, H. Parastar. Recent trends in application of multivariate curve resolution approaches for improving gas chromatography-mass spectrometry analysis of essential oils. Talanta 2011, 85, 835.

[19] A. Salvador, A. Chisvert. Analysis of Cosmetic Products. Elsevier B.V, Amsterdam, 2007.

[20] R. A. Hites. Gas Chromatography Mass Spectrometry. Prentice Hall, Upper Saddle River, NJ, 1997. 